\title{
Contour Patterns of Chinese Intonational Phrases*
}

\author{
CHEN Hu \\ Luoyang University of Foreign Languages, Luoyang, China
}

\begin{abstract}
Due to the interaction between lexical tones and intonation in Chinese speech melody, the contour patterns of Chinese IPs (intonational phrases) have long been an intriguing yet complicated subject. Within the AM (Autosegmental-Metrical) framework, this paper makes an empirical study on the subject based on a small-scale database of 22 standard Chinese news stories. A five-degree $D$ value system is proposed for data normalization so that various contour patterns of IPs can be identified and compared despite complications in pitch register and pitch range. The major findings are: Chinese IPs mainly comprise 2, 3, or 4 ips (intermediate phrases). And the contour patterns of Chinese IPs suggest that the statement intonation in Chinese is primarily indicated by the near-bottom $D_{\min }$ value of the last ip. Meanwhile, when the primary indicator is not typical, the falling trend of the last part of the top-line can serve as a compensatory perceptual clue.
\end{abstract}

Keywords: contour patterns, Chinese IP (intonational phrase), the AM (Autosegmental-Metrical) approach

\section{Introduction}

The intonation of Chinese, a typical tone language, has long been an intriguing subject for prosodic study. Among others, the interaction between lexical tones and discourse intonation lies at the core of the question. To observe the actual resultant contour patterns of Chinese IPs (intonational phrases) in connected speech, this paper makes a data-based survey of CCTV (China Central Television) news in the AM (Autosegmental-Metrical) approach.

\section{Research Method}

According to the AM approach, intonational features of tune and relative prominence are distributed in utterances in ways allowed by the prosodic structure. As the basic unit of intonational pitch contours, an IP, which is often demarcated by boundary markers like pauses, anacrusis, boundary-final lengthening, pitch reset, etc., comprises one or more ips (intermediate phrases). An ip in turn consists of one or more pitch accents and ends with a phrase tone. At the end of the last ip of an IP, there is a boundary tone (CHEN, 2006; Ladd, 1996).

\section{Materials}

The research materials are 22 complete news stories selected from the recording of the half-hour CCTV news program Xinwenlianbo aired on January 1, 2008. Xinwenlianbo remains China's most authoritative official news program and a live model of Putonghua (i.e., Standard Chinese).

\section{Processing and Annotation}

The software Praat (ver. 5112) is used for the processing, annotation, and pitch data extraction of the

\footnotetext{
* Acknowledgements: This research is sponsored by China State Social Sciences Fund (07CYY001). CHEN Hu, associate professor, Ph.D., The English Department, Luoyang University of Foreign Languages.
} 
target materials.

For the purpose of this research, five tiers are annotated for the selected news stories: PY (pinyin), PWs (prosodic words), GS (grammatical structure), ip, and IP.

\section{Data Normalization}

Given that the pitch register and pitch range of an IP vary a lot as its announcer or discourse location or function changes, in order to make the observation of the pitch contours of different IPs comparable, the 5-degree pitch scale is adopted for the normalization of the pitch data extracted from Praat in the unit of Hertz.

Here is the formula (LIN, 2002):

$$
D=5 \times\left(F_{0}-F_{0 \min }\right) \div\left(F_{0 \max }-F_{0 \min }\right)
$$

In the formula, $F_{0}$ refers to the target pitch value to be normalized from $\mathrm{Hz}$ to $D$, while $F_{0 \text { max }}$ and $F_{0 \text { min }}$ refer to the maximum and minimum pitch values of an IP respectively.

All the survey of intonational contour patterns in this paper is made on the basis of the 5-degree $D$ values.

\section{Representation of Contour Patterns}

As mentioned before, Chinese is a typical tone language, whose pitch contours reflect the combination of lexical tones and intonational modification. For a clear and holistic description of the contour patterns of Chinese IPs, we follow the method of observing the respective movement trends of the top-line and bottom-line of the component ips within an IP (SHEN, 1994).

Specifically, to describe the contour pattern of an IP, the maximum and minimum $D$ values of each of its component ip are first calculated based on extracted pitch values; the contour patterns, then, are represented by the series of R's (Rise) and F's (Fall) obtained by successively deducting the $D_{\max }$ or $D_{\min }$ value of a former ip with that of the next one, where a positive result or zero is recorded as a $\mathrm{R}$ while a negative result a $\mathrm{F}$. And the final representation of the contour pattern of each IP is put down as a serial code consisting of two hyphenated parts, each reflecting the overall pitch trend of the top-line and the bottom-line of the IP pitch contour. For instance, the contour pattern RF-FF indicates that the IP consists of three ips, and that its top-line (i.e., the connected line between the $D_{\max }$ values of each pair of neighboring ips) first rises and then falls (RF); meanwhile, its bottom-line forms a consecutive falling trend (FF).

\section{Results}

\section{General Description}

Among the 22 target news stories, there are altogether 156 IPs, 452 ips, 1,389 PWs, and 3,164 syllables.

Table 1

\section{Distribution of IP Groups}

\begin{tabular}{lcc}
\hline Group of IP & Number & Percentage (\%) \\
\hline IP with 1 ip & 5 & 3.2 \\
IP with 2 ips & 62 & 39.7 \\
IP with 3 ips & 59 & 37.8 \\
IP with 4 ips & 21 & 13.5 \\
IP with 5 ips & 6 & 3.9 \\
IP with 6 ips & 2 & 1.3 \\
IP with 7 ips & 1 & 0.6 \\
\hline Total & 156 & 100.0 \\
\hline
\end{tabular}


On average, each news story is made up of 7.1 IPs; each IP comprises 2.9 ips; each ip contains 3.1 PWs; and each PW consists of 2.3 syllables.

IPs are classified into seven groups (as shown in Table 1) according to the number of ips they contain. From Table 1, we can see that the IPs with 2, 3, and 4 ips constitute the overwhelming majority (a total of over 90 percent in our data) of all IPs. Due to space limit, only the survey of the contour patterns of the 2-ip and 3-ip IPs is presented.

\section{Phrasing}

Grammatically, among the total of 156 IPs, 81 (51.9\%) are predicative verb phrases without a subject; 69 (44.2\%) are complete clauses with both subject and predicate; the remaining 6 (3.9\%) belong to others such as stranded adverbial phrases, subject phrases, etc., which all involve relatively complicated internal structures. The majority of the verb phrases as IPs is not unexpected given that Chinese, as a parataxis language, is well-known for its rich use of run-on sentences.

Within the IPs, the phrasing of ips is of much greater variety. Table 2 is the list of the top 10 most frequently recurrent ips.

On the whole, despite occasional exceptions, either the phrasing of the IPs or the ips demonstrates high agreement with grammatical boundaries.

Table 2

Distribution of the Top 10 ips

\begin{tabular}{lcc}
\hline ip & Frequency & Percentage (\%) \\
\hline AV & 25 & 5.5 \\
V & 19 & 4.2 \\
DS & 17 & 3.8 \\
VO & 16 & 3.5 \\
DO & 15 & 3.3 \\
VDO & 15 & 3.3 \\
O & 14 & 3.1 \\
A & 9 & 2.0 \\
DDS & 9 & 2.0 \\
DDO & 8 & 1.8 \\
\hline Total & 147 & 32.5 \\
\hline
\end{tabular}

In Table 2 (and elsewhere of this study), the letters A, V, D, S, and O refer to Adverbial, Verb, Determiner (i.e., modifier of a noun), Subject, and Object respectively.

\section{Relative Prominence}

Previous studies have long indicated that in Chinese intonation, prosodic prominence is highly related, among other factors, to the relative prominence in the realization of the target pitch value ( $\mathrm{H}$ for Tone 1,2 and 4; L for Tone 3) and pitch range of the stressed syllables; meanwhile, GS has a bearing on the distribution of the so-called "logical stress" in Chinese (YE, 2001).

Table 3 shows the average values of the $D_{\max }$ and $D_{\min }$ of the corresponding PWs of the six major Chinese grammatical constituents in our data.

From Table 3, it is clear that the average Determiner has a higher $\mathrm{H}$ value and a higher $\mathrm{L}$ value than the average Subject or Object, which conforms to the existing observation that in an unmarked Determiner-Head 
structure, the relative prominence goes to the Determiner (as indicated by its higher $D_{\max }$ value) whereas the structure as a whole tend to form a single prosodic unit (as indicated by its higher $D_{\min }$ value) (SHEN, 1994). The same is true with the Adverbial-Verb structure, in which the Adverbial enjoys relative prominence while the two still form a single prosodic unit.

Table 3

Feature D Values of the Major Chinese Grammatical Constituents

\begin{tabular}{lll}
\hline Grammatical constituent & $D_{\max }$ & $D_{\min }$ \\
\hline Subject & 3.5 & 0.8 \\
Verb & 3.0 & 0.9 \\
Object & 2.8 & 0.3 \\
Determiner & 3.8 & 1.3 \\
Adverbial & 3.8 & 1.7 \\
Complement & 3.1 & 0.4 \\
\hline
\end{tabular}

It is also noteworthy that the Object and the Complement, whose unmarked positions are IP-final, both have the lowest average $D_{\min }$ values approaching the bottom-line of the 5-degree scale space, a clear indicator of the existence of the statement boundary tone (CHEN, 2006).

\section{Contour Patterns of IPs With 2 ips}

Table 4 shows the frequency and percentage of all the contour patterns of 2-ip IPs in our data. Table 5 shows the average $D_{\max }$ and $D_{\min }$ values of the component ips of each contour pattern in a left-to-right order, from which the overall pitch trend of each contour pattern can be clearly projected.

Table 4

Contour Patterns of 2-ip IPs

\begin{tabular}{llc}
\hline Contour pattern & Frequency & Percentage (\%) \\
\hline F-F & 36 & 58.1 \\
R-F & 12 & 19.3 \\
F-R & 10 & 16.1 \\
R-R & 4 & 6.5 \\
\hline Total & 62 & 100.0 \\
\hline
\end{tabular}

Table 5

Average $D_{\max }$ and $D_{\min }$ Values of the Component ip's of Each 2-ip Contour Pattern

\begin{tabular}{lcccc}
\hline \multirow{2}{*}{ Contour pattern } & \multicolumn{3}{c}{ ip1 } & \multicolumn{2}{c}{ ip2 } \\
\cline { 2 - 5 } & $D_{\max }$ & $D_{\min }$ & $D_{\max }$ & $D_{\min }$ \\
\hline F-F & 5.0 & 0.6 & 3.6 & 0.0 \\
R-F & 4.3 & 0.6 & 5.0 & 0.0 \\
F-R & 5.0 & 0.0 & 3.8 & 0.2 \\
R-R & 4.5 & 0.0 & 5.0 & 0.2 \\
\hline
\end{tabular}

\section{Contour Patterns of IPs With 3 ips}

Table 6 shows the frequency and percentage of all the contour patterns of 3 -ip IPs in our data. Table 7 show the average $D_{\max }$ and $D_{\min }$ values of the component ips of the top six most frequent contour patterns in a left-to-right order, from which the overall pitch trend of each contour pattern can be clearly projected. 
Table 6

Contour Patterns of 3-ip IPs

\begin{tabular}{lcc}
\hline Contour pattern & Frequency & Percentage (\%) \\
\hline RF-RF & 16 & 27.1 \\
RF-FF & 12 & 20.3 \\
RF-FR & 5 & 8.5 \\
FF-FR & 5 & 8.5 \\
RR-RF & 4 & 6.8 \\
FR-RF & 4 & 6.8 \\
FR-FR & 3 & 5.1 \\
FF-RF & 3 & 5.1 \\
RR-FR & 2 & 3.4 \\
FR-FF & 2 & 3.4 \\
RR-FF & 1 & 1.7 \\
FF-RR & 1 & 1.7 \\
FF-FF & 1 & 1.7 \\
\hline Total & 59 & 100.0 \\
\hline
\end{tabular}

Table 7

Average $D_{\max }$ and $D_{\min }$ Values of the Component ip's of the Top Six 3-ip Contour Patterns

\begin{tabular}{lcccccc}
\hline \multirow{2}{*}{ Contour pattern } & \multicolumn{2}{c}{ ip1 } & \multicolumn{3}{c}{ ip2 } & \multicolumn{2}{c}{ ip3 } \\
\cline { 2 - 8 } & $D_{\max }$ & $D_{\min }$ & $D_{\max }$ & $D_{\min }$ & $D_{\max }$ & $D_{\min }$ \\
\hline RF-RF & 3.4 & 0.2 & 5.0 & 0.6 & 3.9 & 0.1 \\
RF-FF & 3.6 & 1.6 & 5.0 & 0.6 & 3.9 & 0.0 \\
RF-FR & 4.7 & 0.3 & 5.0 & 0.0 & 3.3 & 0.1 \\
FF-FR & 5.0 & 0.4 & 4.4 & 0.0 & 3.1 & 0.5 \\
RR-RF & 4.0 & 0.4 & 4.7 & 0.8 & 5.0 & 0.0 \\
FR-RF & 4.7 & 0.7 & 3.6 & 0.8 & 4.7 & 0.0 \\
\hline
\end{tabular}

\section{Discussion}

As is shown in Table 4, all the four logically possible contour patterns of 2-ip IPs are observed in our data. However, their frequency distribution is quite uneven, with the F-F pattern, which features both a falling top-line and a falling bottom-line, accounting for more than a half of the total. If the pitch trends of the top-line and the bottom-line are observed separately, the total frequencies of a falling top-line and a falling bottom-line get even higher. Such observations are in a way quite expected given the existence of declination and downstep and the fact that the IPs in our survey are either statements or parts of statements.

Furthermore, from Table 5, it is clear that in both the F-R and R-R patterns the rising in the bottom-line is very slight, and the $D_{\min }$ values of the ending ips are both quite near the bottom-line of the 5-degree scale. These help to ensure the statement function of the relevant pitch contours.

Things get more complicated in the 3-ip IPs. From Table 5, we can see that as the number of ips grows from two to three, the diversity and variety of contour patterns boom a lot. What adds to the complication is that when observed separately all the four possible patterns can be observed for both the top-line and the bottom-line. Apparently, as shown in Table 7, there seems to be no evident regularity in the performance of the different contour patterns. However, through a careful observation and comparison of the second halves of the top-line and bottom-line of each pitch contour in Table 7, a certain regularity can be found indeed: When the 
ending point of the bottom-line is low (i.e., 0 or near 0 ), the ending point of the top-line can be either high (as in the RR-RF and FR-RF patterns), mid (as in the RF-RF and RF-FF patterns), or low (as in the RF-FR pattern); however, when the ending point of the bottom-line is relatively non-low, the ending point of the top-line must then be relatively low and form a clear falling trend from the former $D_{\max }$ value so as to ensure the perception of the statement boundary tone (as in the FF-FR pattern). The same observation also applies to the contour patterns of 2-ip IPs as shown in Table 5. Therefore, we can come to the tentative conclusion that the near-bottom $D_{\min }$ value of the last ip serves as the primary indicator of the statement intonation in Chinese, while the falling trend of the last part of the top-line can serve as an compensatory auxiliary indicator when the primary indicator is not typical.

\section{Conclusions}

In standard Chinese news materials, on the whole, the phrasing of the IPs and the ips demonstrates high agreement with grammatical boundaries. Among the six major Chinese grammatical constituents, the Determiner and the Adverbial enjoy relative prominence in comparison with the Subject, the Object and the Verb while the Object and the Complement both have near-bottom average $D_{\min }$ values.

The IPs with 2, 3, and 4 ips constitute the majority of all IPs. The contour patterns of all IPs indicate that the near-bottom $D_{\min }$ value of the last ip serves as the primary indicator of the statement intonation in Chinese, while the falling trend of the last part of the top-line can serve as a compensatory auxiliary indicator when the primary indicator is not typical.

\section{References}

CHEN, H. (2006). English and Chinese intonational phonology: A contrastive study. Kaifeng, China: Henan University Press. Cruttenden, A. (2000). Intonation (2nd ed.). Beijing: Peking University Press.

Ladd, D. (1996). Intonational phonology. Cambridge: Cambridge University Press.

LIN, M.-C. (2002). Chinese prosodic structure and functional intonations (Report of phonetic research, Phonetics Laboratory of the Institute of Linguistics, CASS).

Pierrehumbert, J. (1980). The phonology and phonetics of English intonation (Ph.D. thesis, Massachusetts Institute of Technology).

SHEN, J. (1994). Configuration and categories of Chinese intonation. Dialect, (3), 221-228.

SHEN, X.-N. (1990). The prosody of Mandarin Chinese. Berkeley: University of California Press.

YE, J. (2001). The grammatical functions of Chinese discourse prosody. Shanghai: Huadong Normal University Press. 